\title{
Immunohistochemical investigation of cytokine expression levels as biomarkers in transrectal ultrasound-guided needle biopsy specimens of prostate adenocarcinoma
}

\author{
JAGTAR SINGH ${ }^{1,2}$, THANUJA THACHIL ${ }^{3}$, MATHEW SUJI EAPEN ${ }^{2}$, AIJYE LIM $^{4}$, WAJIHA SUFYAN ${ }^{4}$, \\ ROBERT RAWSON ${ }^{4}$, HENRY DUNCAN $^{5}$, PAOLO DE IESO $^{6}$ and SUKHWINDER SINGH SOHAL ${ }^{2}$ \\ ${ }^{1}$ College of Health and Human Sciences, Charles Darwin University, Northern Territory 0810; \\ ${ }^{2}$ Department of Laboratory Medicine, School of Health Sciences, College of Health and Medicine, \\ University of Tasmania, Launceston, Tasmania 7248; ${ }^{3}$ Ballarat Austin Radiation Oncology Centre, Victoria 3350; \\ ${ }^{4}$ Department of Anatomical Pathology, Royal Darwin Hospital 0810; ${ }^{5}$ Urology Department, Darwin Private Hospital, \\ Northern Territory 0810; ${ }^{6}$ Peter MacCallum Cancer Centre, Victoria 3000, Australia
}

Received October 10, 2020; Accepted June 24, 2021

DOI: $10.3892 / \mathrm{mco} .2021 .2353$

\begin{abstract}
Cytokines influence the biological behaviour of prostate cancer (PC) and may influence patient outcome and serve as useful prognostic biomarkers. The aim of the present study was to evaluate cytokine expression levels in prostatic needle biopsy specimens and the association with clinicopathological characteristics of patients with PC. A total of 18 patients with PC who underwent transrectal ultrasound (TRUS) guided prostate biopsy were included in the clinical study. These patients were naïve to radiotherapy (RT) or androgen deprivation therapy prior to TRUS biopsy and clinical follow up data was collected. Cytokine expression levels were analysed by using immunohistochemistry and Spearman's correlation test was used to determine the correlation between cytokine expression and clinicopathological characteristics. Expression levels of pro-inflammatory TNF- $\alpha$ and IL- 6 decreased as Gleason score (GS) increased; however, a statistically significant difference was not detected. A statically significant correlation was observed between needle biopsy specimen and pre-RT plasma sample expression levels of pro-inflammatory TNF- $\alpha$ and IL-6 ( $\mathrm{P}=0.01$ and $\mathrm{P}=0.05$, respectively) and anti-inflammatory TGF- $\beta 1(\mathrm{P}=0.05)$. However, further studies are needed to confirm these results using a larger sample size to confirm the prognostic value of pro-inflammatory TNF- $\alpha$ and IL-6 and anti-inflammatory TGF- $\beta 1$ in patients with PC.
\end{abstract}

Correspondence to: Dr Jagtar Singh, College of Health and Human Sciences, Charles Darwin University, Ellengowan Drive, Casuarina, Northern Territory 0810, Australia

E-mail: cmarkersp534e@gmail.com

Key words: cytokine expression, prostate cancer, immunohistochemical staining, prostatic needle biopsy specimen

\section{Introduction}

Prostate cancer (PC) is the second most commonly diagnosed malignancy (excluding non-melanoma skin cancer) and the fifth leading cause of cancer-associated mortality in men worldwide in 2018 (1). Overall, 1.28 million men were diagnosed with PC (accounting for $15 \%$ of all cancer cases in men) in 2018 , with $\sim 70 \%$ of cases $(759,000)$ occurring in developed countries, including the United States of America, Australia, New Zealand and Europe (1). In current medical practice, prognostic markers for PC include serum prostate-specific antigen (PSA) levels, tumour Gleason score (GS) and clinical tumour grading (2). Predictive accuracy may be improved by introducing better biomarkers into clinical practice (3). Previously, molecular biomarkers such as TNF- $\alpha$, IL-1, IL-6, cyclin $\mathrm{E}$ and metallothionein-2A, have been evaluated for their efficiency in predicting disease progression, response to therapy and survival in patients with PC (4-7).

Certain pro-inflammatory cytokines, including IL-6, TNF- $\alpha$, IL-1 and IL-17, serve an essential role in radiotherapy (RT) resistance and enable tumour progression, invasion and angiogenesis (7-9). Cytokines are water-soluble, low molecular weight proteins that transport signals between cells (10). Rubin et al (11) were among the first to describe the role of cytokines in mediating RT-induced toxicity: They reported that levels TGF- $\beta$, IL- 1 and TNF- $\alpha$ increase immediately following RT exposure and that elevated TGF- $\beta$ levels are associated with increased risk of pulmonary fibrosis (11). Christensen et al (12) reported that interferon- $\gamma($ IFN- $\gamma)$ and IL-6 levels are significantly increased during prostate RT and are associated with increased acute gastrointestinal and genitourinary toxicity.

In addition to higher serum PSA levels and other preliminary assessments, histopathological investigations of PC in needle biopsy specimens predict tumour behaviour and assist with therapeutic decision-making (13). In clinical practice, the pathology report of PC includes the grade of tissue differentiation according to GS and a quantitative 
assessment of tumour volume per biopsy in either length in mm or percentage of a tumour $(14,15)$. GS is based on the histological pattern of arrangement of carcinoma cells in hematoxylin-stained prostatic tissue (16). The final GS is obtained by summing of pattern-numbers of the primary and secondary tissue grade, ranging from 2 to 10 (16). GS quantifies pathological aggressiveness and is also one of the key factors in treatment decision-making, together with TNM staging, age and pre-treatment blood PSA levels (17). However, histological examination has several limitations, such as morphological mimics of prostate carcinoma, including adenosis (a non-cancerous condition), atypical adenomatous hyperplasia and very low- or high-grade carcinoma, which hinder the interpretation of tumour biopsy $(13,18)$.

The present clinical study evaluated expression levels of pro-inflammatory TNF- $\alpha$ and IL- 6 and anti-inflammatory TGF- $\beta 1$ in prostatic needle biopsy and blood plasma specimens. The study also aimed to analyse the correlation between pro-inflammatory TNF- $\alpha$ and IL- 6 and TGF- $\beta 1$ expression levels with GS, pre-operative serum PSA and pre-RT plasma cytokine levels.

\section{Materials and methods}

Patients and clinical data. Between July 2015 and April 2016, a total of 18 male patients with PC were recruited at Alan Walker Cancer Care Centre (Darwin, Australia) for this prospective clinical study. Eligible patients were $\geq 18$ years old, had histologically confirmed prostate adenocarcinoma and Eastern Cooperative Oncology Group performance status of 0 to 1 and had not received prior prostate surgery. Exclusion criteria included metastatic disease at presentation, prior history of malignancy (excluding non-melanoma skin cancer) and serious illness precluding safe administration of RT. These patients were naïve to RT or androgen deprivation therapy (ADT) before transrectal ultrasound (TRUS) biopsy and clinical data were collected. All PC cases were classified into as follows: Low-[clinical (c)T stage $\leq 2 \mathrm{a}$; PSA $<10 \mathrm{ng} / \mathrm{ml}$; GS $\leq 6]$; intermediate-(cT=2b; PSA, 10-20 ng/ml; GS=7) and high-risk (cT $\geq 2 \mathrm{c} ;$ PSA $>20 \mathrm{ng} / \mathrm{ml} ; \mathrm{GS}=8-10$ ) (2). The present study was approved by the Human Research and Ethics Committee of the Northern Territory (approval no. 2015-2385) Department of Health and Menzies School of Health Research. Written informed consent was obtained from all participants to provide access to prostate tissue biopsies, blood samples collected at various time intervals before, during and after therapy and medical and pathology records from Royal Darwin Hospital and Alan Walker Cancer Care Centre.

Immunohistochemistry (IHC) staining. Tissue samples were fixed in $10 \%$ formalin overnight at room temperature before being embedded in paraffin. The tissue was sectioned to $4 \mu \mathrm{m}$ and mounted on poly-lysine-coated slides (Dako; Agilent Technologies, Inc.). All tissue sections were stored in a $50^{\circ} \mathrm{C}$ water bath. Slides were dried for $30 \mathrm{~min}$ in a thermostat at $60^{\circ} \mathrm{C}$. All sections were deparaffinised using xylene and subsequently rehydrated with a series of graded ethanol dilutions. Then, antigen retrieval was performed by placing slides in a Coplin jar with target retrieval solution (Dako; Agilent Technologies, Inc.; $\mathrm{pH}, 9.0$ ) for $20 \mathrm{~min}$ at $90-95^{\circ} \mathrm{C}$ in a hot water bath. All sections were marked using a Dako PEP pen (Agilent Technologies, Inc.) for accuracy.

Sections were incubated in methanol containing 3\% hydrogen peroxide for $30 \mathrm{~min}$ at room temperature and washed twice (3 min/wash) with TBS washing buffer. Goat serum (Dako; Agilent Technologies, Inc.) was applied to all sections and incubated at room temperature for $10 \mathrm{~min}$. Primary antibodies (Novus Biologicals, LLC) were used to determine expression levels of pro-inflammatory TNF- $\alpha$ and IL-6 and TGF- $\beta 1$ in tumour biopsy samples from patients with PC. All tissues were incubated at room temperature for $1 \mathrm{~h}$ using the following primary antibodies: Anti-mouse monoclonal TNF- $\alpha$ (1:50; cat. no. NB600-1422) and TGF- $\beta 1$ (1:100; cat. no. NBP2-22114SS) and anti-rabbit polyclonal IL-6 (1:100; cat. no. NB600-1131SS). Antibody diluent was substituted with primary antibody for negative control sections (Dako; Agilent Technologies, Inc.). All tissue sections were rinsed in TBS as aforementioned. Then, 3-4 drops of secondary antibody (REAL Link-biotinylated secondary Ab2; cat. no. K5001; Dako; Agilent Technologies, Inc.) were applied to all tissue sections and incubated for $10 \mathrm{~min}$ at room temperature. All tissue sections were rinsed twice with TBS then incubated at room temperature with horseradish peroxidase-conjugated streptavidin for $10 \mathrm{~min}$ by adding 3-4 drops to the slides (Dako; Agilent Technologies, Inc.). Finally, all sections were developed with 3'-diaminobenzidine for $5 \mathrm{~min}$ at room temperature and counterstained with Mayer's haematoxylin at room temperature for another $2 \mathrm{~min}$. All tissue sections were dehydrated via a graded series of ethanol dilutions and washed with xylene. After staining, coverslips were applied and sealed using permanent mounting medium.

Microscopic analysis. IHC-stained slides were evaluated for expression of pro-inflammatory TNF- $\alpha$ and IL- 6 and TGF- $\beta 1$ by light microscopy (magnification, $\mathrm{x} 20$ ) in a blinded manner by two clinical pathology consultants. Expression levels of pro-inflammatory TNF- $\alpha$ and IL- 6 and TGF- $\beta 1$ were evaluated using a semi-quantitative scale based on the proportion of positive-stained cells as follows:,$-<10 ;+, 10-50 ;++, 51-80$; ,$+++>80 \%(6,19,20)$.

ELISA. Levels of pro-inflammatory TNF- $\alpha$ and IL- 6 and TGF- $\beta 1$ in pre-RT plasma were assessed. For plasma cytokine analysis, ELISA kits were used including human TNF- $\alpha$ (cat. no. KAC1751), TGF- $\beta 1$ (cat. no. EHTGFBI), IL-6 (cat. no. KAC1261) and IL-8 (cat. no. KAC1301; all Thermo Fisher Scientific, Inc.). Assay kits were chromogen-based and cytokine concentration (colour) was quantified using a Titertek Multiskan MCC/340 plate reader according to the manufacturer's instructions. Each assay was calibrated against a standard curve with a full range predetermined for each cytokine and sample source.

Statistical analysis. Statistical analysis was performed using GraphPad Prism 7 software (GraphPad Software, Inc.). Established clinical variables included in the study were age, pre-operative PSA, risk stratification, c and pathological (p) TNM stage and GS. Data are presented as the mean \pm SD $(n=18)$. Spearman's correlation test was performed to assess the correlation between pro-inflammatory TNF- $\alpha$ and IL-6 
Table I. Clinicopathological characteristics of patients with prostate cancer.

\begin{tabular}{|c|c|c|}
\hline Characteristic & $\mathrm{N}$ & Percentage, $\%$ \\
\hline Age, years $($ mean $\pm S D)$ & $66.83 \pm 7.93(53.00-80.00)$ & \\
\hline \multicolumn{3}{|l|}{ Pre-operative PSA, ng/ml } \\
\hline$<10$ & 6.00 & 33 \\
\hline $10-20$ & 9.00 & 50 \\
\hline$>20$ & 3.00 & 17 \\
\hline \multicolumn{3}{|l|}{ Risk stratification } \\
\hline Low & 0.00 & 0 \\
\hline Intermediate & 5.00 & 28 \\
\hline High & 13.00 & 72 \\
\hline \multicolumn{3}{|l|}{ cTNM stage } \\
\hline T1a-cNOM0 & 4.00 & 23 \\
\hline T2a-cN0M0 & 6.00 & 33 \\
\hline T3a-cN0M0 & 8.00 & 44 \\
\hline \multicolumn{3}{|l|}{ pTNM stage } \\
\hline T1a-cN0M0 & 0.00 & 0 \\
\hline T2a-cNOMO & 16.00 & 89 \\
\hline T3a-cN0M0 & 2.00 & 11 \\
\hline \multicolumn{3}{|l|}{ Gleason score } \\
\hline 6 & 1.00 & 6 \\
\hline 7 & 8.00 & 44 \\
\hline $8-10$ & 9.00 & 50 \\
\hline
\end{tabular}

c, clinical; p, pathological; PSA, prostate-specific antigen.

and anti-inflammatory TGF- $\beta 1$ expression levels and the aforementioned variables. Spearman's correlation test was also used to determine the linear correlation between cytokine expression levels in pre-RT plasma and corresponding prostatic needle biopsy specimens. $\mathrm{P}<0.05$ was considered to indicate a statistically significant difference.

\section{Results}

Clinical characteristics of patients with PC. The clinicopathological information of participants, such as age, pre-operative PSA levels, risk stratification, c and pTNM stage, and GS, are summarised in Table I.

The mean age of patients with $\mathrm{PC}$ at the time of diagnosis was 66.83 \pm 7.93 years (range, 53.00-80.00 years). The mean pre-operative serum PSA levels were $16.03 \pm 15.81 \mathrm{ng} / \mathrm{ml}$ (range, $4.00-71.00 \mathrm{ng} / \mathrm{ml}) ; 33 \%$ of patients (6/18) exhibited PSA $<10.00,50 \%(9 / 18)$ exhibited 10.00-20.00 ng/ml PSA and $17 \%(3 / 18)$ exhibited PSA $>20.00 \mathrm{ng} / \mathrm{ml}$. The mean GS was $7.88 \pm 1.14$ (range, $6.00-10.00$ ); $6 \%$ of patients $(1 / 18)$ exhibited $\mathrm{GS}=6.00,44 \%(8 / 19)$ exhibited $\mathrm{GS}=7.00$ and $50 \%$ (9/18) exhibited GS=8.00-10.00. Tumour staging was divided into c and pT stage. For cT stage, $23 \%$ of patients (4/18) were T1a-c, 33\% (6/18) were T2a-c and $44 \%(8 / 18)$ were T3a-c. For pT stage, $0 \%$ of patients $(0 / 18)$ were T1a-c, $89 \%(16 / 18)$ were T2a-c and $11 \%(2 / 18)$ were T3a-c. Only one patient $(6 \%)$ exhibited one ipsilateral pelvic node involved before therapy.
Cytokine expression in prostatic needle biopsy specimens. IHC staining for pro-inflammatory TNF- $\alpha$, IL- 6 and anti-inflamatory TGF- $\beta 1$ revealed elevated levels of these cytokines in most tumour tissue samples compared with healthy tissue (Figs. 1 and 2). Malignant prostate cells exhibited brown cytoplasmic staining, indicating expression of pro-inflammatory TNF- $\alpha$ and IL- 6 and TGF- $\beta 1$ in prostatic needle biopsy specimens from patients with PC.

Correlation between cytokine expression and pre-operative serum PSA levels. Serum PSA is used as a guide to initiate prostatic biopsies and to monitor men older than 50 years for PC (21). Serum PSA level is the most commonly used tumour biomarker for PC There was no correlation between expression levels of pro-inflammatory TNF- $\alpha$ and IL-6 and anti-inflammatory TGF- $\beta 1$ and pre-operative serum PSA levels (data not shown).

Correlation between cytokine expression levels and GS. Spearman's correlation test was performed to assess the association between cytokine expression levels and GS. GS ranges from 1-5 and describes how much cancer from a biopsy resembles healthy (lower score) or abnormal tissue (higher score). Most cancers score $\geq 3$ in anatomical pathology practice depending on aggressiveness (22). Figs. 1 and 2 show $\mathrm{H} \& \mathrm{E}$ and IHC staining in biopsy samples with GS as follows: $3+3=6,3+4=7,4+3=7,4+4=8,4+5=9,5+4=9$ and $5+5=10$. Lower expression levels of pro-inflammatory TNF- $\alpha$ and 


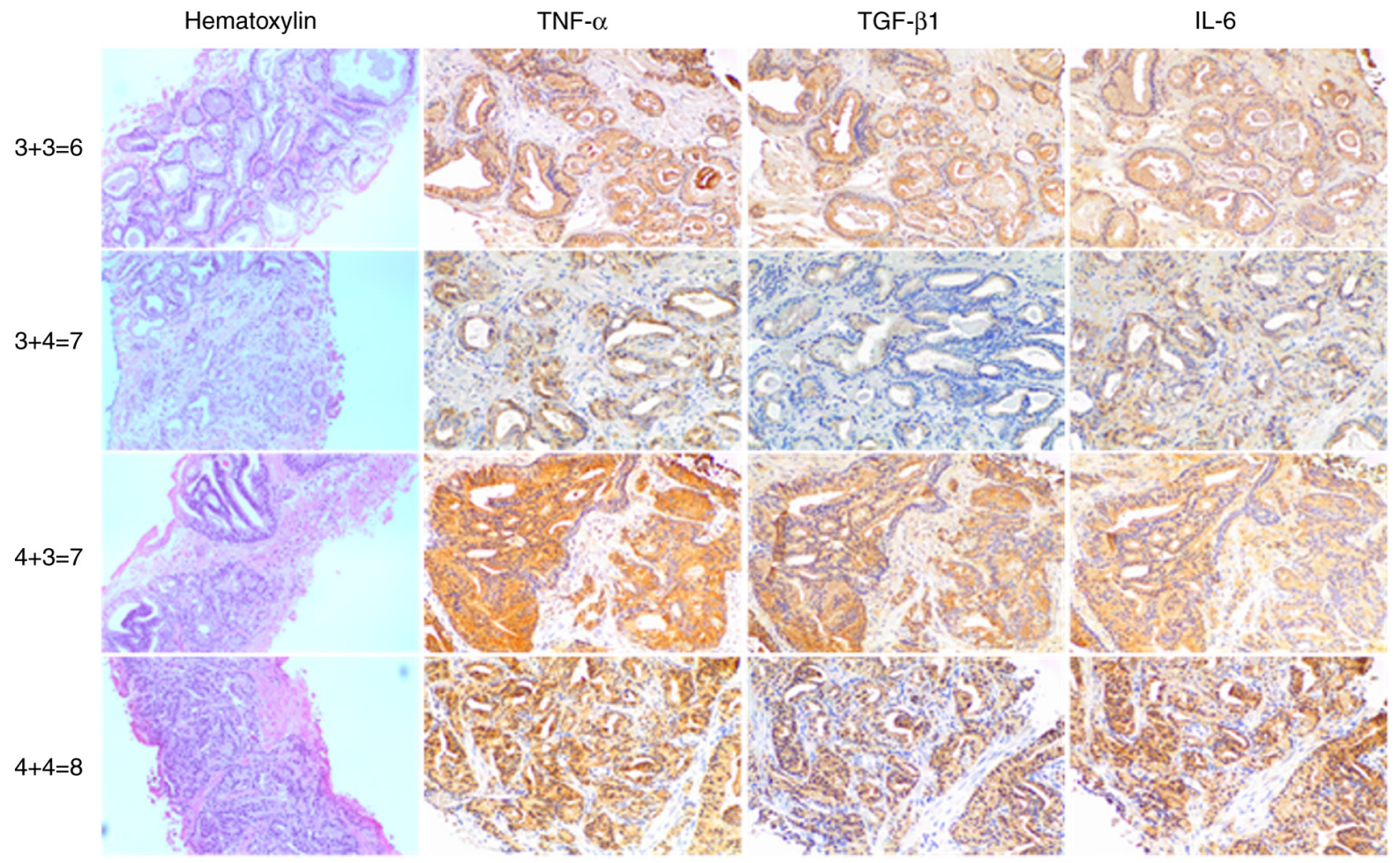

Figure 1. Hematoxylin and immunohistochemical staining with TNF- $\alpha$, TGF- $\beta 1$ and IL-6 antibodies in prostatic needle biopsy specimens from patients with prostate cancer with Gleason score as follows: $3+3=6,3+4=7,4+3=7$ and $4+4=8$. Magnification, $x 20$.

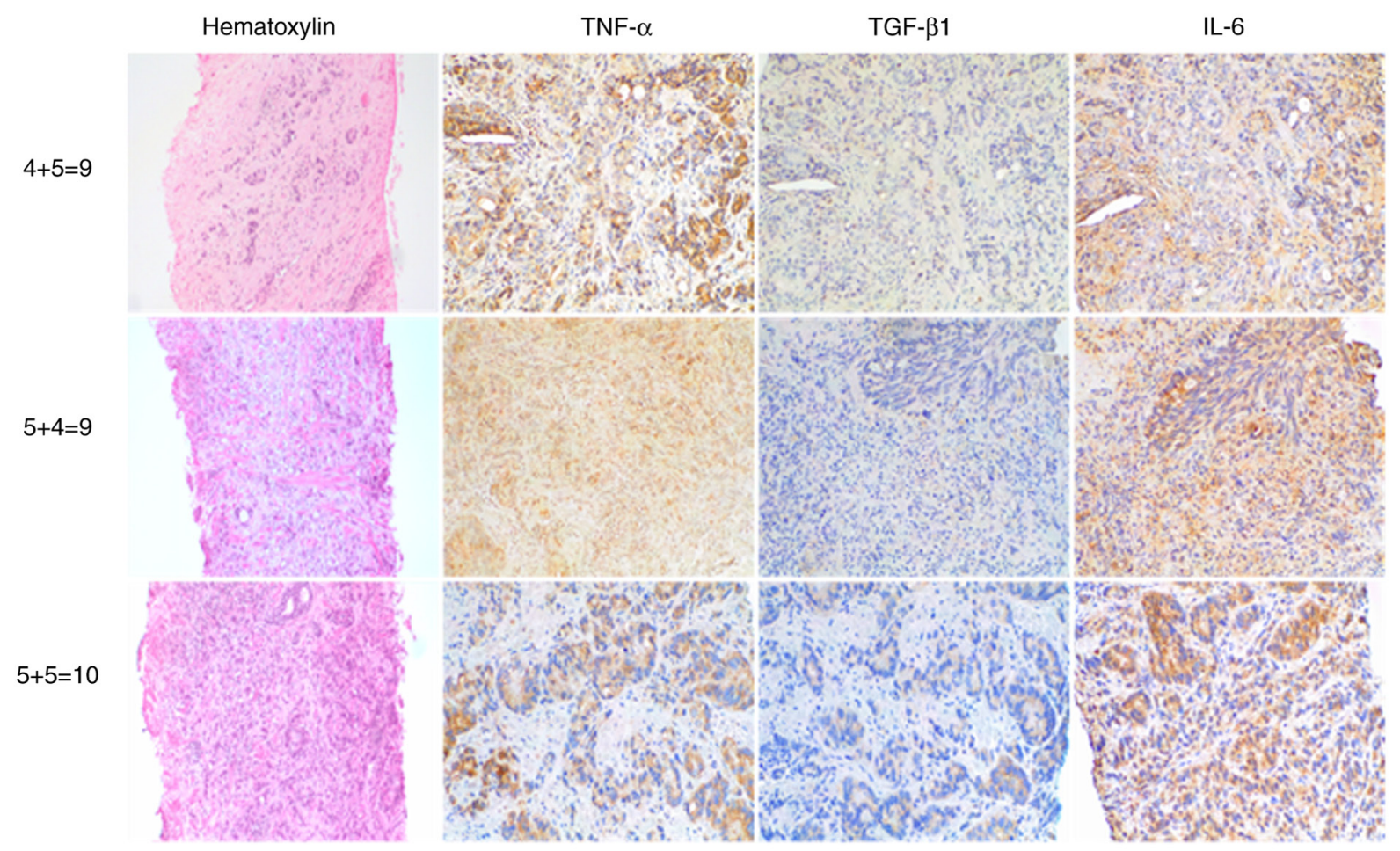

Figure 2. Hematoxylin and immunohistochemical staining with TNF- $\alpha$, TGF- $\beta 1$ and IL-6 antibodies in prostatic needle biopsy specimens from patients with prostate cancer with Gleason score as follows: $4+5=9,5+4=9$ and 5+5=10. Magnification, $\mathrm{x} 20$.

IL-6 were associated with high GS; however, no statistically significant association was found (TNF- $\alpha, \rho=-0.5096$; IL-6, $\rho=-0.3169$; Fig. 3). Anti-inflammatory TGF- $\beta$ did not show any association with GS $(\rho=-0.3241)$.
Correlation between cytokine expression levels in biopsy samples and pre-RT plasma. The present study evaluated the potential impact of tumour-derived cytokine production on circulating plasma levels. IHC expression levels of 

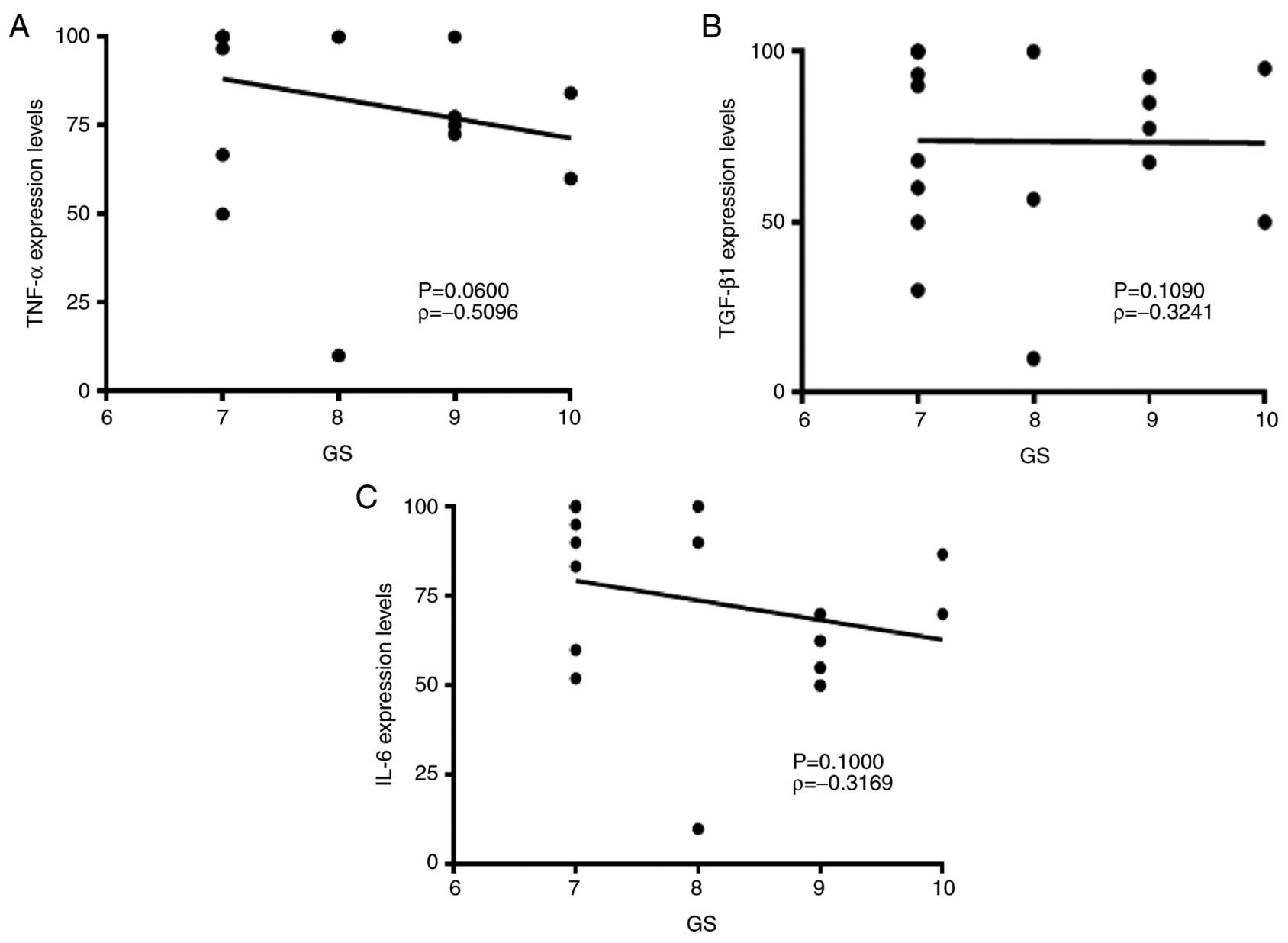

Figure 3. Analysis of expression levels of TNF- $\alpha$, TGF- $\beta 1$ and IL-6 and GS in patients with prostate cancer. Correlation between expression levels of (A) TNF- $\alpha$, (B) TGF- $\beta 1$ and (C) IL-6 and GS. GS, Gleason score.

pro-inflammatory TNF- $\alpha$ and IL-6 and anti-inflammatory TGF- $\beta 1$ were increased in prostatic needle-biopsy specimens with increased pre-RT plasma cytokines levels detected by ELISA (Fig. 4). A statistically significant association was found between staining intensity of proinflammatory TNF- $\alpha$ and IL- 6 and anti-inflammatory TGF- $\beta 1$ in prostatic needle biopsy specimens and concentration in pre-RT plasma (TNF- $\alpha$, $\rho=0.7629$; TGF- $\beta 1, \rho=0.5742$; IL-6, $\rho=0.5294$; Fig. 4).

\section{Discussion}

Histopathological analysis and GS can predict outcomes of PC (23). A number of clinical studies have reported the significance of novel biomarkers that may be used in future as predictors of prognosis and tumour development $(24,25)$. Numerous biomarkers, such as cytokines, hormone receptors, oncogenes and tumour suppressor genes, are well-established in clinical scientific literature (26). The role of pro-inflammatory cytokines, including TNF- $\alpha$, IL-1 or IL- 6 , in cancer development has been established in PC $(26,27)$.

The primary clinical challenge in PC is the lack of diagnostic tests, including PSA screening and histopathological grading, to differentiate between aggressive and indolent tumours (28). PSA is present in normal prostatic secretions and its levels are often elevated in patients with PC $(29,30)$. Rodriguez-Berriguete et al demonstrated an association between elevated stromal expression of IL-1 receptor-associated kinase 1 (IRAK-1) and high pre-operative serum PSA levels. IL-1 $\beta$ expression in PC tumours and IL-1 receptor, type II and IRAK-1 expression levels in tumour stroma have prognostic value after adjusting for the effects of $\mathrm{pT}$ stage, GS and total pre-operative serum PSA (6). There is a significant association between positive p27 expression and lower mean serum PSA levels ( $\mathrm{P}=0.091)$ (31). Shariat et al (32) reported that pre-treatment serum levels of TGF- $\beta 1$, IL- 6 and soluble IL-6 receptor levels are positively correlated with pre-operative PSA levels ( $\mathrm{P}=0.004, \mathrm{P}<0.001$ and $\mathrm{P}=0.011$, respectively). Also, patients with elevated expression of IL-1 $\alpha$ exhibit higher serum PSA levels (>20 ng/ml) (33). In the present clinical study, no association between expression levels of pro-inflammatory TNF- $\alpha$ and IL- 6 and TGF- $\beta 1$ in prostatic needle biopsy specimens and pre-operative serum PSA levels was detected.

GS histopathological grading is an important prognostic indicator of PC $(34,35)$. GS quantifies pathological aggressiveness of PC and is one of the principal factors in treatment decision-making, along with TNM stage, age and presenting PSA levels. GS of 8-10 represents a clinically aggressive form of the disease and is used to classify patients as high-risk (36). High-grade cancer poses increased risk of biochemical, locoregional and distant recurrence with subsequent detrimental effects on overall survival (36). Michalaki et al (37) demonstrated that serum levels of IL-6 
A

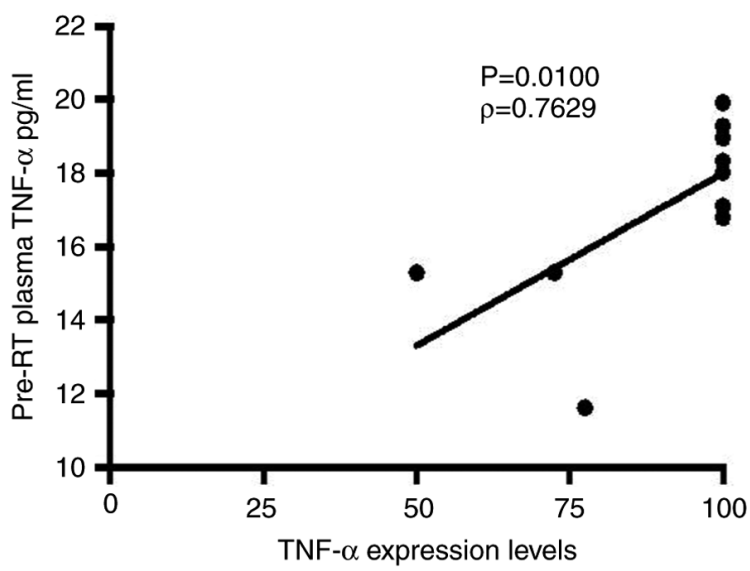

C
$\mathrm{B}$
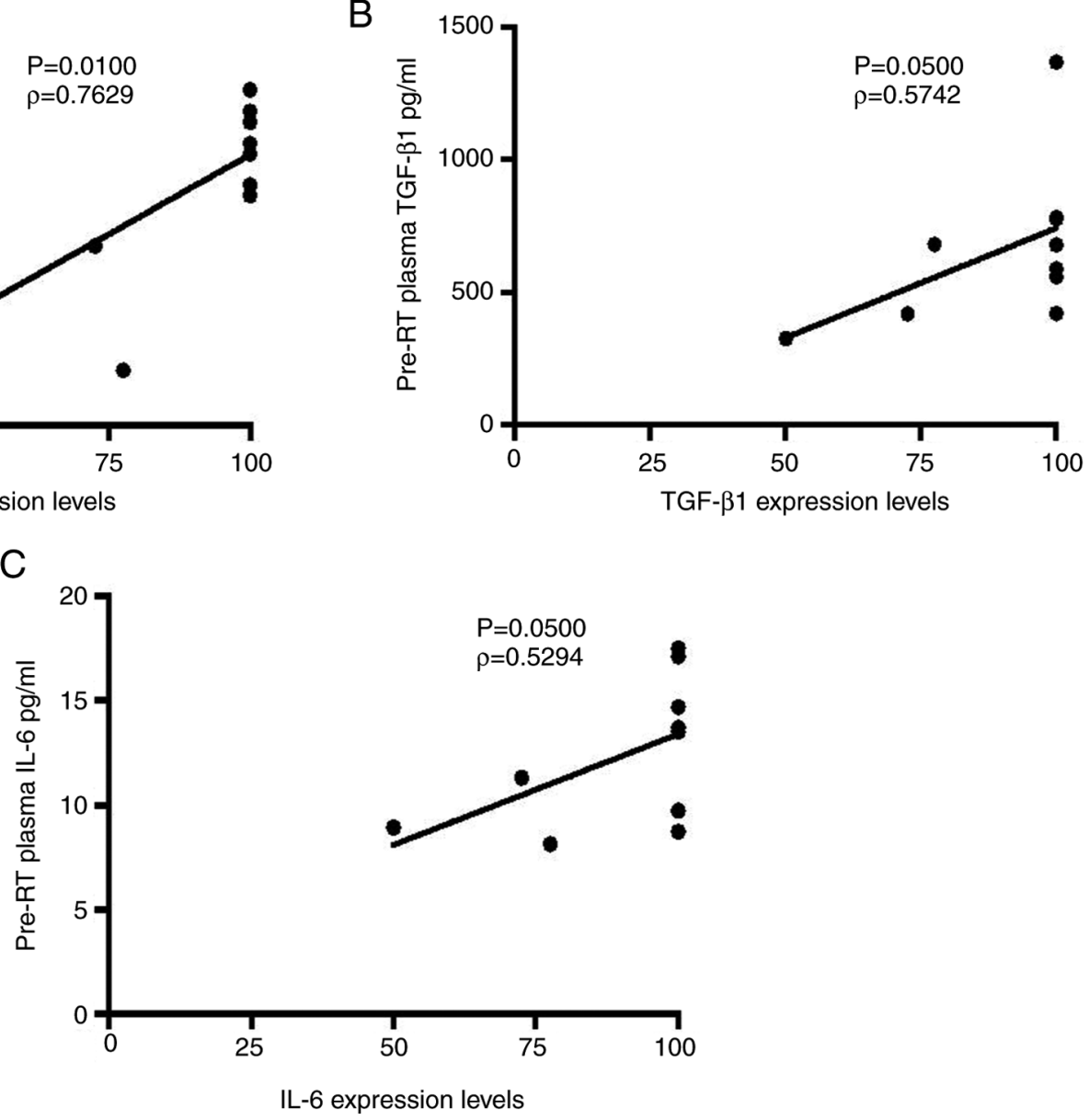

Figure 4. Analysis of cytokine expression levels of TNF- $\alpha$, TGF- $\beta 1$ and IL-6 in biopsy samples and pre-RT plasma from patients with prostate cancer. Correlation between (A) TNF- $\alpha$, (B) TGF- $\beta 1$ and (C) IL-6 expression levels in biopsy samples and pre-RT plasma. RT, radiotherapy.

are significantly higher in patients with metastatic disease and GS>6. Another clinical study reported that elevated levels of IL- 6 are associated with GS $\geq 7$ and metastases in regional lymph nodes (32). Gomes et al (38) reported that high six-transmembrane epithelial antigen of the prostate 1 (STEAP1) expression is significantly associated with GS=7-9; patients with higher GS (7-9) exhibited elevated STEAP1 expression, whereas those with lower GS (5-6) showed moderate STEAP1 expression. The data in current study are opposite to those of the aforementioned studies: We identified lower expression levels of pro-inflammatory TNF- $\alpha$ and IL- 6 were associated with high GS, however, this was not statistically significant.

The present clinical study also evaluated pro-inflammatory TNF- $\alpha$ and IL- 6 and TGF- $\beta 1$ plasma cytokine levels in patients with PC. Rube et al (39) reported a statistically significant correlation between pre-RT plasma IL- 6 and TGF- $\beta 1$ cytokine levels and staining intensity of corresponding tumour biopsy. The present study revealed elevated levels of pro-inflammatory TNF- $\alpha$ and IL- 6 and TGF- $\beta 1$ in prostatic needle biopsy specimens of patients with increased pre-RT plasma cytokine levels using ELISA. Furthermore, a statistically significant correlation was detected between IHC staining intensity of pro-inflammatory TNF- $\alpha$ and IL- 6 and TGF- $\beta 1$ in prostatic needle biopsy specimens and expression levels in pre-RT plasma (TNF- $\alpha, \mathrm{P}=0.01$; TGF- $\beta 1, \mathrm{P}=0.05$ and $\mathrm{IL}-6, \mathrm{P}=0.05$ ).
Our previous study demonstrated pre-RT plasma cytokine expression levels in patients with PC. Further experiments will investigate correlation between cytokine expression in prostatic needle biopsy specimens and concentration in pre-RT plasma (40).

The present clinical study identified a correlation between cytokine expression levels in biopsy samples with GS and pre-RT plasma cytokine levels. However, a statistically significant difference was only found between pre-RT plasma and biopsy sample cytokine levels. Further clinical studies are required to validate these findings and identify biomarkers in the clinical setting to predict patient outcomes and improve treatment success.

\section{Acknowledgements}

The authors would like to thank Ms Beryl Edward (Anatomical Pathology Department) and Ms Ruby Hilario (Royal Darwin Hospital) for assistance with tissue selection and sectioning for IHC analysis and clinical data collection.

\section{Funding}

The present clinical study was supported by grants from the College of Health and Human Sciences, Charles Darwin University, Northern Territory, Australia. 


\section{Availability of data and materials}

All data generated or analyzed during this study are included in this published article.

\section{Authors' contributions}

JS, SSS, TT, HD and PDI designed the study, collected and analysed patient data and wrote the manuscript. JS, TT, HD and PDI recruited patients and collected blood and tissue samples. AL, WS and RR interpreted and graded the IHC images. MSE and SSS statistically analysed experimental data. All authors read and approved the final manuscript. JS and SSS confirm the authenticity of all the raw data.

\section{Ethics approval and consent to participate}

The present study was approved by the Human Research and Ethics Committee of the Northern Territory (approval no. 2015-2385) and Department of Health and Menzies School of Health Research. Written informed consent was obtained from all participants.

\section{Patient consent for publication}

Not applicable.

\section{Competing interests}

The authors declare that they have no competing interests.

\section{References}

1. Mattiuzzi C and Lippi G: Current cancer epidemiology. J Epidemiol Glob Health 9: 217-222, 2019.

2. D'Amico AV, Whittington R, Malkowicz SB, Schultz D, Blank K Broderick GA, Tomaszewski JE, Renshaw AA, Kaplan I, Beard CJ and Wein A: Biochemical outcome after radical prostatectomy, external beam radiation therapy, or interstitial radiation therapy for clinically localized prostate cancer. JAMA 280: 969-974, 1998

3. Burke HB: Predicting clinical outcomes using molecular biomarkers. Biomark Cancer 8: 89-99, 2016.

4. Milicevic N, Mrcela M, Galic J and Marjanovic K: Expression of proinflammatory cytokine interleukin-6 in tissue samples of human prostate obtained by needle biopsy. Pathol Res Pract 211 : 865-870, 2015

5. MaD,Zhou Z, Yang B,He Q,Zhang Q and Zhang XH: Association of molecular biomarkers expression with biochemical recurrence in prostate cancer through tissue microarray immunostaining. Oncol Lett 10: 2185-2191, 2015.

6. Rodriguez-Berriguete G, Sanchez-Espiridion B, Cansino JR, Olmedilla G, Martinez-Onsurbe P, Sanchez-Chapado M, Paniagua R, Fraile B and Royuela M: Clinical significance of both tumor and stromal expression of components of the IL-1 and TNF- $\alpha$ signaling pathways in prostate cancer. Cytokine 64 555-563, 2013.

7. Deorukhkar A and Krishnan S: Targeting inflammatory pathways for tumor radiosensitization. Biochem Pharmacol 80 : 1904-1914, 2010

8. Steiner GE, Newman ME, Paikl D, Stix U, Memaran-Dagda N, Lee $\mathrm{C}$ and Marberger MJ: Expression and function of pro-inflammatory interleukin IL-17 and IL-17 receptor in normal, benign hyperplastic, and malignant prostate. Prostate 56: 171-182, 2003.

9. Poutahidis T, Rao VP, Olipitz W, Taylor CL, Jackson EA, Levkovich T, Lee CW, Fox JG, Ge Z and Erdman SE: CD4 ${ }^{+}$ lymphocytes modulate prostate cancer progression in mice. Int J Cancer 125: 868-878, 2009.
10. George DJ, Halabi S, Shepard TF, Sanford B, Vogelzang NJ, Small EJ and Kantoff PW: The prognostic significance of plasma interleukin-6 levels in patients with metastatic hormone-refractory prostate cancer: Results from cancer and leukemia group B 9480. Clin Cancer Res 11: 1815-1820, 2005.

11. Rubin P, Johnston CJ, Williams JP, McDonald S and Finkelstein JN: A perpetual cascade of cytokines postirradiation leads to pulmonary fibrosis. Int J Radiat Oncol Biol Phys 33: 99-109, 1995.

12. Christensen E, Pintilie M, Evans KR, Lenarduzzi M, Menard C, Catton CN, Diamandis EP and Bristow RG: Longitudinal cytokine expression during IMRT for prostate cancer and acute treatment toxicity. Clin Cancer Res 15: 5576-83, 2009.

13. Hoogland AM, Kweldam CF and van Leenders GJ: Prognostic histopathological and molecular markers on prostate cancer needle-biopsies: A review. Biomed Res Int 2014: 341324, 2014.

14. Epstein JI, Allsbrook WC Jr, Amin MB and Egevad LL; ISUP Grading Committee: The 2005 International society of urological pathology (ISUP) consensus conference on gleason grading of prostatic carcinoma. Am J Surg Pathol 29: 1228-1242, 2005.

15. Van der Kwast T, Bubendorf L, Mazerolles C, Raspollini MR, Van Leenders GJ, Pihl CG and Kujala P: Guidelines on processing and reporting of prostate biopsies: The 2013 update of the pathology committee of the European randomized study of screening for prostate cancer (ERSPC). Virchows Arch 463: 367-377, 2013.

16. Gleason DF: Histologic grading of prostate cancer: A perspective. Hum Pathol 23: 273-279, 1992.

17. Tagai EK, Miller SM, Kutikov A, Diefenbach MA, Gor RA, Al-Saleem T, Chen DYT, Fleszar S and Roy G: Prostate cancer patients' understanding of the gleason scoring system: Implications for shared decision-making. J Cancer Educ 34: 441-445, 2019.

18. Epstein JI, Feng Z, Trock BJ and Pierorazio PM: Upgrading and downgrading of prostate cancer from biopsy to radical prostatectomy: Incidence and predictive factors using the modified Gleason grading system and factoring in tertiary grades. Eur Urol 61: 1019-1024, 2012.

19. Zimmermann AK, Camenisch U, Rechsteiner MP, Bode-Lesniewska B and Rossle M: Value of immunohistochemistry in the detection of BRAF(V600E) mutations in fine-needle aspiration biopsies of papillary thyroid carcinoma. Cancer Cytopathol 122: 48-58, 2014

20. Kapoor P and Deshmukh R: VEGF: A critical driver for angiogenesis and subsequent tumor growth: An IHC study. J Oral Maxillofac Pathol 16: 330-337, 2012.

21. Wu YS, Wu XB, Zhang N, Jiang GL, Yu Y, Tong SJ, Jiang HW, Mao SH, Na R and Ding Q: Evaluation of PSA-age volume score in predicting prostate cancer in Chinese population. Asian J Androl 20: 324-329, 2018.

22. Pessoa R, Werahera PN and Kim FJ: Chapter 102-Prostate Cancer. In Abernathy's Surgical Secrets, 7th edition. Harken AH and Moore EE (eds). Elsevier, Philadelphia, PA, pp450-451, 2018

23. Kir G, Seneldir H and Gumus E: Outcomes of Gleason score $3+4=7$ prostate cancer with minimal amounts $(<6 \%)$ vs. $\geq 6 \%$ of Gleason pattern 4 tissue in needle biopsy specimens. Ann Diagn Pathol 20: 48-51, 2016.

24. Freedland SJ and Moul JW: Prostate specific antigen recurrence after definitive therapy. J Urol 177: 1985-1991, 2007.

25. Doganavsargil B, Simsir A, Boyacioglu H, Cal C and Hekimgil M: A comparison of p21 and p27 immunoexpression in benign glands, prostatic intraepithelial neoplasia and prostate adenocarcinoma. BJU Int 97: 644-648, 2006.

26. Pfitzenmaier J, Vessella R, Higano CS, Noteboom JL, Wallace D Jr and Corey E: Elevation of cytokine levels in cachectic patients with prostate carcinoma. Cancer 97: 1211-1216, 2003.

27. Mauri D, Pentheroudakis G, Tolis C, Chojnacka M and Pavlidis N: Inflammatory prostate cancer: An underestimated paraneoplastic clinical manifestation. Urol Oncol 23: 318-322, 2005.

28. Romero Otero J, Garcia Gomez B, Campos Juanatey F and Touijer KA: Prostate cancer biomarkers: An update. Urol Oncol 32: 252-260, 2014.

29. Wu D, Ni J, Beretov J, Cozzi P, Willcox M, Wasinger V, Walsh B, Graham P and Li Y: Urinary biomarkers in prostate cancer detection and monitoring progression. Crit Rev Oncol Hematol 118: 15-26, 2017.

30. Lilja H, Ulmert D and Vickers AJ: Prostate-specific antigen and prostate cancer: Prediction, detection and monitoring. Nat Rev Cancer 8: 268-278, 2008. 
31. Nassif AE and Tambara Filho R: Immunohistochemistry expression of tumor markers CD34 and P27 as a prognostic factor of clinically localized prostate adenocarcinoma after radical prostatectomy. Rev Col Bras Cir 37: 338-344, 2010 (In English, Portuguese).

32. Shariat SF, Kattan MW, Traxel E, Andrews B,Zhu K, Wheeler TM and Slawin KM: Association of pre- and postoperative plasma levels of transforming growth factor beta(1) and interleukin 6 and its soluble receptor with prostate cancer progression. Clin Cancer Res 10: 1992-1999, 2004.

33. Cansino Alcaide JR, Vera San Martin R, Rodriguez de Bethencourt Codes F, Bouraoui Y, Rodriguez Berriguete G, Oueslati R, Perez-Utrilla M, De la Pena Barthel J, Paniagua Gomez-Alvarez R and Royuela Garcia M: Prostatic specific antigen (PS), pro-inflammatory cytokines, and prostatic pathology (benign prostatic hyperplasia and cancer). Relationship with malignancy. Arch Esp Urol 62: 359-366, 2009 (In Spanish).

34. Epstein JI: An update of the Gleason grading system. J Urol 183 : 433-440, 2010

35. Shen MM and Abate-Shen C: Molecular genetics of prostate cancer: New prospects for old challenges. Genes Dev 24: 1967-2000, 2010.
36. Stock RG, Cesaretti JA, Hall SJ and Stone NN: Outcomes for patients with high-grade prostate cancer treated with a combination of brachytherapy, external beam radiotherapy and hormonal therapy. BJU Int 104: 1631-1636, 2009.

37. Michalaki V, Syrigos K, Charles P and Waxman J: Serum levels of IL-6 and TNF-alpha correlate with clinicopathological features and patient survival in patients with prostate cancer. Br J Cancer 90: 2312-2316, 2004.

38. Gomes IM, Arinto P, Lopes C, Santos CR and Maia CJ: STEAP1 is overexpressed in prostate cancer and prostatic intraepithelial neoplasia lesions, and it is positively associated with Gleason score. Urol Oncol 32: 53.e23-29, 2014.

39. Rube CE, Palm J, Erren M, Fleckenstein J, Konig J, Remberger K and Rube C: Cytokine plasma levels: reliable predictors for radiation pneumonitis? PLoS One 3: e2898, 2008.

40. Singh J, Sohal SS, Ahuja K, Lim A, Duncan H, Thachil T and De Ieso P: Levels of plasma cytokine in patients undergoing neoadjuvant androgen deprivation therapy and external beam radiation therapy for adenocarcinoma of the prostate. Ann Transl Med 8: 636, 2020. 